\title{
On the Beta Function for Anisotropic Current Interactions in 2D
}

\author{
B. Gerganov ${ }^{a}$, A. LeClair ${ }^{a, b}$ and M. Moriconi ${ }^{a}$ \\ ${ }^{a}$ Newman Laboratory, Cornell University, Ithaca, NY 14853 \\ and \\ ${ }^{b}$ LPTHE, 4 Place Jussieu, Paris, France.
}

\begin{abstract}
By making use of current-algebra Ward identities we study renormalization of general anisotropic current-current interactions in 2D. We obtain a set of algebraic conditions that ensure the renormalizability of the theory to all orders. In a certain minimal prescription we compute the $\beta$ eta function
\end{abstract} to all orders.

Left-right current-current interactions in 2D arise in many physical systems, for example in the KosterlitzThouless transitions and in the study of free electrons in random potentials. The chiral Gross-Neveu model is the simplest example which is symmetry preserving (isotropic) [1]. Because such interactions are marginal in $2 \mathrm{D}$, the couplings are dimensionless and there is usually no small parameter to expand in order to explore strong coupling.

In this work we determine the $\beta$ eta function to all orders in a certain minimal prescription. This should be sufficient for the study of fixed points. The primary tool that allows us to isolate the log divergences at arbitrary order are the Ward identities for the currents. Kutasov performed a similar computation in the simpler isotropic case (i.e. non-abelian Thirring model, which is equivalent to the chiral Gross-Neveu model) but argued his result was the leading order in $1 / k$ where $k$ is the level of the current algebra [4]. We believe our result to be exact.

We do not expect that there is anything dramatically new to learn for the isotropic case. The behavior of models like the Gross-Neveu model is very well understood. This is in contrast to the anisotropic, i.e. symmetry breaking perturbations where one can expect richer phenomena. In the anisotropic case not all perturbations are in fact renormalizable. We find three renormalizability conditions that ensure the theory is renormalizable to all orders. Given these conditions are satisfied, we find a compact expression for the summation of all orders in perturbation theory.

We know of no reason to expect additional nonperturbative corrections to the $\beta$ eta function due for instance to instantons. Our result should be a useful tool for exploring strong coupling physics for the many models in this class. In this Letter we only report the main result and defer applications to future publications.

Consider a conformal field theory with Lie algebra symmetry realized in the standard way [2,3]. It possesses left and right conserved currents, $J^{a}(z), \bar{J}^{a}(\bar{z})$, where $z=x+i y, \bar{z}=x-i y$, satisfying the operator product expansion (OPE)

$$
J^{a}(z) J^{b}(0) \sim \frac{k \eta^{a b}}{z^{2}}+\frac{1}{z} f_{c}^{a b} J^{c}(0)+\ldots,
$$

and similarly for $\bar{J}^{a}(\bar{z}) . \eta^{a b}$ in the above equation is a metric (Killing form) and $k$ is the level.

We include the case of Lie superalgebras with applications to disordered systems in mind. Here each current $J^{a}$ has a grade $[a]=0$ or 1 , and the tensors satisfy: $\eta^{a b}=$ $(-)^{[a][b]} \eta^{b a}, f^{a b c}=-(-)^{[b][c]} f^{a c b}, f_{c}^{a b}=-(-)^{[a][b]} f_{c}^{b a}$, where $f^{a b c}=f_{i}^{a b} \eta^{i c}$. For superalgebras, $\eta^{a b}$ is generally not diagonalizable, but we have $\eta_{a b} \eta^{b c}=\delta_{a}^{c}$.

The conformal field theory can be perturbed by marginal operators which are bilinear in the currents. The most general action is

$$
S=S_{\mathrm{cft}}+\int \frac{d^{2} x}{2 \pi} \sum_{A} g_{A} \mathcal{O}^{A}, \mathcal{O}^{A}=\sum_{a, \bar{a}} d_{a \bar{a}}^{A} J^{a} \bar{J}^{\bar{a}}
$$

where $S_{\mathrm{cft}}$ is the conformal field theory with the currentalgebra symmetry, and $d_{a \bar{a}}^{A}$ are certain tensors that define the model. The simplest case is that of a single coupling where $d_{a \bar{a}}=\eta_{a \bar{a}}$ so that the perturbation is built on the Casimir and preserves the symmetry.

For these models the $\beta$ eta function beyond 2 loops is prescription dependent. Let $\beta_{g}=b_{2} g^{2}+b_{3} g^{3}+\ldots$ The prescription dependence is equivalent to a redefinition of the coupling $g^{\prime}=g^{\prime}(g)$. Let $g^{\prime}=g+c g^{2}+\ldots$. One easily sees that $\beta^{\prime}\left(g^{\prime}\right)=b_{2} g^{\prime 2}+b_{3} g^{\prime 3}+\ldots$, so that the one- and two-loop coefficients $b_{2}$ and $b_{3}$ are universal. Though the higher loop contributions are prescription dependent, the existence of fixed points is not: if $\beta_{g}=0$, then $\beta^{\prime}\left(g^{\prime}\right)=$ $\frac{\partial g^{\prime}}{\partial g} \beta_{g}=0$. For this reason, the particular prescription we will adopt is meaningful.

We will compute the $\beta$ eta function in the following way. Let $X$ be any arbitrary product of fields and suppose we isolate the ultra-violet logarithmic divergences in the following way:

$$
\langle X\rangle=\sum_{A} F_{A}(g, \log a) \int \frac{d^{2} x}{2 \pi}\left\langle\mathcal{O}^{A}(x) X\right\rangle+\ldots .
$$

where $a$ is a short distance cutoff (lattice spacing). In general $F_{A}$ has an expansion in powers of $\log a$ :

$$
F_{A}=-a_{A}^{(0)}(g)+\sum_{n=1}^{\infty} a_{A}^{(n)}(g) \log ^{n} a .
$$

Requiring $d F_{A} / d \log a=0$ gives

$$
\beta_{g_{A}} \frac{d g_{A}}{d \log a}=G_{A B}^{-1} a_{B}^{(1)}, \quad G_{A B}=\partial_{g_{B}} a_{A}^{(0)} .
$$


In our models equation (3) holds due to the Ward identities for the currents. This makes the computation much simpler that the two-loop computations in perturbed conformal field theory carried out in [5]. The order $g^{n}$ term in the expansion is

$$
\begin{aligned}
& \frac{(-)^{n}}{n !} g_{A_{1}} \ldots g_{A_{n}} d_{a_{1} \bar{a}_{1}}^{A_{1}} \ldots d_{a_{n} \bar{a}_{n}}^{A_{n}} \\
& \quad \times \int d 1 \ldots d n\left\langle J^{a_{1}} \bar{J}^{\bar{a}_{1}}(1) \ldots J^{a_{n}} \bar{J}^{\bar{a}_{n}}(n) X\right\rangle,
\end{aligned}
$$

where $\int d 1=\int d^{2} x_{1} / 2 \pi$. The Ward identities read

$$
\begin{aligned}
& \left\langle J^{a_{1}}(1) \ldots J^{a_{n}}(n) X\right\rangle \\
& =\sum_{i \neq 1} \frac{k \eta^{a_{1} a_{i}}}{z_{1 i}^{2}}\left\langle J^{a_{2}}(2) \ldots \widehat{J}^{a_{i}}(i) \ldots J^{a_{n}}(n) X\right\rangle \\
& \quad+\frac{f_{c}^{a_{1} a_{i}}}{z_{1 i}}\left\langle J^{a_{2}}(2) \ldots J^{c}(i) \ldots J^{a_{n}}(n) X\right\rangle,
\end{aligned}
$$

where $z_{i j}=z_{i}-z_{j}$, and $\widehat{J}$ means the current is removed. (We do not display the factors of $(-)^{[a][b]}$ in the above equation and eq. (9) below.) In order to compute $F_{A}$ one only needs the integrals

$$
\begin{gathered}
\int \frac{d^{2} \rho}{2 \pi} \frac{1}{\rho-w} \frac{1}{\bar{\rho}-\bar{z}}=-\log |w-z|, \\
\int \frac{d^{2} \rho}{2 \pi} \frac{1}{(\rho-w)^{2}} \frac{1}{\bar{\rho}-\bar{z}}=-\frac{1}{2} \frac{1}{w-z}, \\
\int \frac{d^{2} \rho}{2 \pi} \frac{1}{(\rho-w)^{2}} \frac{1}{(\bar{\rho}-\bar{z})^{2}}=\frac{\pi}{2} \delta^{(2)}(w-z),
\end{gathered}
$$

to systematically reduce (6) to one integral. In performing this reduction, we throw away two kinds of contributions: (i) vacuum bubbles proportional to the volume $\int d^{2} x$; (ii) $\log a$ divergences that factorize into a lower order contribution to $a^{(1)}$ times a finite part which is cancelled by dividing by $G_{A B}$. The remaining $\log a$ divergences have a rather simple structure. Consider doing the integral $\int d 1$ in (6) using (7). One is left with 3 kinds of terms $\left[k^{2}\right],[k f]$, and $\left[f^{2}\right]$ terms:

$$
\begin{aligned}
{\left[k^{2}\right]=\frac{\pi k^{2}}{2} } & \int d 2 \ldots d n \sum_{i, j} \delta^{(2)}\left(z_{i j}\right) \eta^{a_{1} a_{i}} \eta^{\bar{a}_{1} \bar{a}_{j}} \\
& \times\left\langle\ldots \widehat{J} \bar{J}^{\bar{a}_{i}}(i) \ldots J^{a_{j}} \widehat{\bar{J}}(j) \ldots X\right\rangle \\
{[k f]=- } & \frac{k}{2} \int d 2 \ldots d n \sum_{i, j}\left\{\eta^{a_{1} a_{i}} f_{\bar{c}}^{\bar{a}_{1} \bar{a}_{j}} \frac{1}{z_{i j}}\right. \\
& \left.\times\left\langle\ldots \widehat{J} \bar{J}^{\bar{a}_{i}}(i) \ldots J^{a_{j}} \bar{J}^{\bar{c}}(j) \ldots X\right\rangle+z \rightarrow \bar{z}\right\} \\
{\left[f^{2}\right]=} & -\int d 2 \ldots d n \sum_{i, j} \log \left|z_{i j}\right| f_{c}^{a_{1} a_{i}} f_{\bar{c}}^{\bar{a}_{1} \bar{a}_{j}} \\
& \times\left\langle\ldots J^{c} \bar{J}^{\bar{a}_{i}}(i) \ldots J^{a_{j}} \bar{J}^{\bar{c}}(j) \ldots X\right\rangle
\end{aligned}
$$

Log $a$ divergences arise in the following ways: (i) The $\left[f^{2}\right]$ term gives a log divergence when the remaining integrals give $\delta^{(2)}\left(z_{i j}\right)$ by repeatedly using $(8 c) .\left(\log \left|z_{i j}\right|=\log a\right.$ when $i=j$, (ii) Repeatedly using (8b) in the $[k f]$ terms can flip $1 / z$ to $1 / \bar{z}$ or vice versa a number of times. Ultimately, a Ward identity involving another $f$ will give a $\log \left(z_{i j}\right)$ like in the $f^{2}$ term. Then again, the remaining integrations must be proportional to $\delta^{(2)}\left(z_{i j}\right)$ to give a $\log a$ divergence.

Integrations of $\log \left(z_{i j}\right)$ with other integrands which are not $\delta$-functions give rise to higher powers of $\log a$ but are not important for the $\beta$-function. We checked explicitly at two loops that the $\log ^{2} a$ divergences are squares of one-loop divergences.

The above contributions can be represented graphically, which will be useful for determining their tensor structure. Let solid lines denote the $J(z)$ OPEs and dashed lines denote the $\bar{J}(\bar{z})$ ones. Denote the $f$-OPEs with arrows. For the holomorphic OPEs for instance:

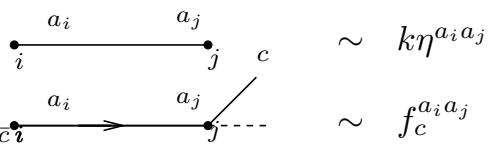

FIG. 1. Diagramatic representation of $k$ - and $f$-OPEs.

First consider $n=$ even, which corresponds to an odd number of loops. There are two types of contributions: Type 2A, 2B. Type 2A is shown graphically in Figure 2:

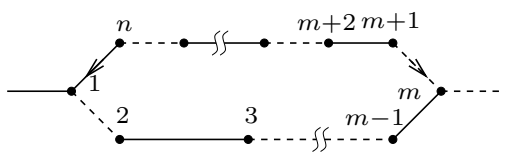

FIG. 2. Type 2A diagram.

The diagrams represent successive integrations from top to bottom. The top alternating holomorphic and antiholomorphic lines represent succession of $1 / z$ to $1 / \bar{z}$ flips using $(8 b)$. The bottom alternating lines represent $\delta$ functions coming from $(8 c)$. The rest of the diagram represents the $f_{c}^{a b} f_{\bar{c}}^{\bar{a} \bar{b}}$ leading to the log divergence coming from $(8 a)$. The external lines represent the leftover $J \bar{J}$ in (3). In the Type $2 \mathrm{~A}$ diagrams, $m$ is odd, $m=1,3, \ldots, n-1$, and there are $n / 2$ diagrams of this type. The Type $2 \mathrm{~B}$ diagrams are distinguished by having the current that remains after the $f_{c}^{a b} \mathrm{OPE}$ becoming an internal part of the diagram:

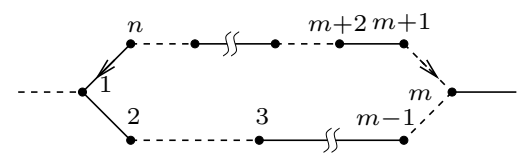

FIG. 3. Type 2B diagram.

Again $m$ is odd, $m=3,5, \ldots, n-1$. There are $(n-2) / 2$ diagrams of this type. 
At $n=$ odd order, diagrams involve two holomorphic $f$ 's or two anti-holomorphic $f$ 's. There are again 2 types, 1A, 1B. The $1 \mathrm{~A}$ diagrams are

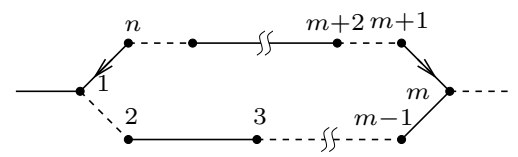

FIG. 4. Type 1A diagram.

Here $m=$ odd $=3,5, \ldots, n-2$. There are $(n-3) / 2$ of these. A Type 1B diagram is distinguished by both $f$ 's coming to the same point:

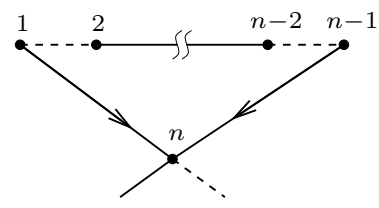

FIG. 5. Type 1B diagram.

There is one diagram of this type. It is the only contribution at two loops. For $n$ odd each of the above diagrams has a " $z / \bar{z}$ relative" where dashed lines are replaced with solid and vice versa. These give the same contribution. The total number of diagrams is then $(n-1)$ for $n$ even or odd.

Each diagram represents a tensor built out of $\eta^{a b}$ and $f_{c}^{a b}$ which must be contracted with $d_{a b}^{A}$ 's. An arbitrary choice of $d_{a b}^{A}$ does not lead to a renormalizable theory, i.e. (3) does not hold. At one loop one needs:

$$
(-)^{[b][c]} d_{a b}^{A} d_{c d}^{B} f_{i}^{a c} f_{j}^{b d}=\sum_{C} C_{C}^{A B} d_{i j}^{C}
$$

for some structure constants $C_{C}^{A B}$. This closure of the operator algebra of $\mathcal{O}^{A}$ is equivalent to the $\mathrm{OPE}$

$$
\mathcal{O}^{A}(z, \bar{z}) \mathcal{O}^{B}(0) \sim \frac{1}{z \bar{z}} C_{C}^{A B} \mathcal{O}^{C}(0)
$$

and it is well-known that $C_{C}^{A B}$ determine the $\beta$ eta function to one loop [7]. This one-loop condition does not ensure renormalizability at higher loops. Systematically examining the higher loop corrections we find that one also needs the quadratic form $d^{A}$ to satisfy

$$
\begin{aligned}
\eta^{i j} d_{a i}^{A} d_{b j}^{B} & =\sum_{C} D_{C}^{A B} d_{b a}^{C} \\
d_{i j}^{A} f_{k}^{j a} f_{b}^{i k} & =\sum_{B} R_{B}^{A} \eta^{a c} d_{c b}^{B}
\end{aligned}
$$

The conditions (10) and (13) guarantee that the theory is renormalizable to all orders. The conditions (13) already arise at 2 loops. One can show from the defining relations that $D, C$ satisfy $D_{C}^{A B}=D_{C}^{B A}, C_{C}^{A B}=$ $C_{C}^{B A}, D_{D}^{A C} D_{E}^{D B}=D_{D}^{A B} D_{E}^{D C}$.
The structure constants $D, R$ also have a meaning in terms of OPEs. Define $T^{A}(z)$ as

$$
T^{A}(z)=d_{a b}^{A} J^{a}(z) J^{b}(z)
$$

suitably normal ordered. $T^{A}$ is a kind of energy-momentum tensor built out of $d_{a b}^{A}$ and the left-moving currents only. In the symmetric case $T^{A}$ is the affine-Sugawara stress-tensor up to a normalization. Then one finds

$$
T^{A}(z) \mathcal{O}^{B}(0) \sim \frac{1}{z^{2}}\left(2 k D_{C}^{A B}+R_{E}^{A} D_{C}^{B E}\right) \mathcal{O}^{C}(0),
$$

In practice this is the most efficient way to compute the $\mathrm{RG}$ data. The $D$ term is distinguished from the $R D$ term by being proportional to $k$. As shown below, the $\beta$ eta function depends only on the combination $R D$ so there is no need to compute $R$ separately. The conditions under which $T^{A}$ actually define a Virasoro algebra [6] are stronger than our renormalizability conditions.

Finally we can write down the beta function. Let us arrange the couplings into a row vector $g=\left(g_{1}, g_{2}, \ldots\right)$. It is convenient to define a matrix of couplings $D(g)$, $D(g)_{B}^{A}=\sum_{C} D_{B}^{A C} g_{C}$. Then $g D^{n}(g)$ is also a row vector. The 4 kinds of contributions (coming from the Type $2 \mathrm{~A}$ through Type 1B diagrams respectively) are

$$
\begin{aligned}
& \beta_{g_{A}}^{(2 \mathrm{~A})}=-\frac{1}{2}\left(\frac{k}{2}\right)^{n-2}\left(g D^{m-1}\right)_{B}\left(g D^{n-m-1}\right)_{C} C_{A}^{B C} \\
& \beta_{g_{A}}^{(2 \mathrm{~B})}=-\frac{1}{2}\left(\frac{k}{2}\right)^{n-2}\left(g D^{m-3}\right)_{B}\left(g D^{n-m-1}\right)_{C} C_{E}^{B C}\left(D^{2}\right)_{A}^{E} \\
& \beta_{g_{A}}^{(1 \mathrm{~A})}=\left(\frac{k}{2}\right)^{n-2}\left(g D^{m-2}\right)_{B}\left(g D^{n-m-1}\right)_{C} C_{E}^{B C} D_{A}^{E} \\
& \beta_{g_{A}}^{(1 \mathrm{~B})}=-\left(\frac{k}{2}\right)^{n-2}\left(g D^{n-2} R D\right)_{A},
\end{aligned}
$$

where $D=D(g)$. (Each diagram comes with a symmetry factor of $n ! / 2$.)

Remarkably, the series can be summed in a closed form. To display the result in a compact way, for any two vectors $v^{1}$ and $v^{2}$ let $C\left(v^{1}, v^{2}\right)$ denote a new row vector, $C\left(v^{1}, v^{2}\right)_{A}=\sum_{B, C} v_{B}^{1} v_{C}^{2} C_{A}^{B C}$. Defining $g^{\prime}=g\left(1-k^{2} D^{2}(g) / 4\right)^{-1}$, then

$$
\begin{aligned}
\beta_{g}=-\frac{1}{2} C\left(g^{\prime},\right. & \left.g^{\prime}\right)\left(1+k^{2} D^{2} / 4\right) \\
+ & \frac{k^{3}}{8} C\left(g^{\prime} D, g^{\prime} D\right) D-\frac{k}{2} g^{\prime} D R D,
\end{aligned}
$$

where again $D=D(g)$. The above $\beta$ eta function determines how couplings flow with increasing length scale $a$, where large $a$ corresponds to the low-energy limit.

The simplest possible case corresponds to a single coupling with $d_{a b}=\eta_{a b}$. Normalizing the Lie algebra generators in the fundamental representation $F$ so that $\operatorname{Tr}\left(t^{a} t^{b}\right)$ $=c_{F} \delta^{a b}=\eta^{a b}$ and using $\eta_{i j} f_{k}^{j c} f_{d}^{i k}=C_{\text {adj }} \delta_{d}^{c}$, one finds

$$
\beta_{g}=\frac{1}{2} \frac{C_{\mathrm{adj}} g^{2}}{\left(1+k g c_{F} / 2\right)^{2}} .
$$


This agrees with previous two-loop calculations for the Gross-Neveu model [8]. (The usual convention corresponds to $c_{F}=1 / 2$ with $k=1$.) A three-loop computation was performed in 9 .

From the result (18) we see that the $\beta$ eta function is identically zero if $C_{\text {adj }}=0$. This occurs for the superalgebras $\operatorname{osp}(2 n+2 \mid 2 n)$ and $\operatorname{PSL}(n \mid n)$ 10 12]. In the work [12], an all orders $\beta$ eta function was proposed in the case of $g l(1 \mid 1)$ using a simple scaling argument, and the result had a structure of the kind described here.

The renormalizability conditions (11, 15) are rather restrictive. Consider for instance a completely anisotropic perturbation $\sum_{a} g_{a} J^{a} \bar{J}^{a}=\sum_{a} g_{a} \mathcal{O}^{a}$ in a basis where $\eta^{a b}=\delta^{a b}$, i.e. the couplings $g_{a}, g_{b}$ are not equal for $a \neq b$. This is not renormalizable in general since the operator algebra of $\mathcal{O}^{a}$ does not close:

$$
\mathcal{O}^{a}(z, \bar{z}) \mathcal{O}^{b}(0) \sim \frac{1}{z \bar{z}} \sum_{c, d} f_{c}^{a b} f_{d}^{a b} J^{c} \bar{J}^{d}(0)
$$

The algebra only closes if $\left[t^{a}, t^{b}\right]$ is proportional to a single generator, since then $f_{c}^{a b} f_{d}^{a b} \propto \delta_{c d}$ and one has $C_{c}^{a b}=\left(f_{c}^{a b}\right)^{2}$. Adopting a basis for $J^{a}$ based on the root system of the Lie algebra, in order to build a renormalizable theory one needs to include additional interactions of the form $(\alpha \cdot H)\left(\alpha^{\prime} \cdot \bar{H}\right)$ where $\alpha, \alpha^{\prime}$ are roots and $H$ is in the Cartan basis. The resulting theory is renormalizable and one can then compute the RG data $C, D, R$ and the $\beta$ eta function. Interesting models which are still renormalizable can be obtained by equating subsets of the couplings consistent with a global sub-symmetry.

The simplest example is $s u(2)$ since it only has one Cartan generator. Let us illustrate our main result in this example. Let the currents be normalized as

$$
\begin{gathered}
J_{3}(z) J^{ \pm}(0) \sim \pm \frac{1}{z} J^{ \pm}(0), J^{+}(z) J^{-}(0) \sim \frac{k}{2} \frac{1}{z^{2}}+\frac{1}{z} J_{3}(0), \\
J_{3}(z) J_{3}(0) \sim \frac{k}{2} \frac{1}{z^{2}} .
\end{gathered}
$$

This corresponds to $\eta^{a b}=\frac{1}{2} \delta^{a b}, C_{\text {adj }}=2$, and $c_{F}=1 / 2$. Consider the interaction

$$
\sum_{A} g_{A} \mathcal{O}^{A}=g_{\perp}\left(J^{+} \bar{J}^{-}+J^{-} \bar{J}^{+}\right)+g_{\|} J_{3} \bar{J}_{3} .
$$

Using (11) and (15) the RG data are: $D_{\perp}^{\perp \perp}=D_{\|}^{\|\| \|}=1 / 2$, $C_{\perp}^{\perp \|}=C_{\perp}^{\| \perp}=-1, C_{\|}^{\perp \perp}=-2,(R D)_{\perp}^{\perp \perp}=(R D)_{\perp}^{\| \perp}=1$, $(R D)_{\|}^{\perp \|}=2$. After some algebra one finds

$$
\begin{aligned}
\beta_{g_{\perp}} & =\frac{g_{\perp}\left(g_{\|}-k g_{\perp}^{2} / 4\right)}{\left(1-k^{2} g_{\perp}^{2} / 16\right)\left(1+k g_{\|} / 4\right)} \\
\beta_{g_{\|}} & =\frac{g_{\perp}^{2}\left(1-k g_{\|} / 4\right)^{2}}{\left(1-k^{2} g_{\perp}^{2} / 16\right)^{2}} .
\end{aligned}
$$

When $g_{\perp}=g_{\|}$one recovers (18).
One can use these $\beta$ eta functions to study KosterlitzThouless flows [13] at strong coupling, or the issue of symmetry restoration recently addressed in [14]. One observes flows to the isotropic line $g_{\perp}=g_{\|}$in the regime $0<g_{\perp, \|}<4 / k$. However there are other features which will be reported elsewhere, along with applications of the above results to disordered fermions [15].

We hope to publish a longer version of this paper with more examples [16].

\section{ACKNOWLEDGMENTS}

We would like to thank M. Ameduri, P. Argyres, Z. Bassi, D. Bernard, V. Dotsenko, and P. Wiegmann for discussions. This work is in part supported by the NSF.

[1] D. Gross and A. Neveu, Phys. Rev. D10 (1974) 3235.

[2] V.G. Knizhnik and A. B. Zamolodchikov, Nucl. Phys. B247 (1984) 83;

[3] E. Witten, Commun. Math. Phys. 92 (1984) 455.

[4] D. Kutasov, Phys. Lett. B227 (1989) 68.

[5] V. Dotsenko, M. Picco, and P. Pujol, Nucl. Phys. B455 (1995) 701, hep-th/9501017.

[6] M. B. Halpern, E. Kiritsis, N. A. Obers and K. Clubok, Phys.Rept. 265 (1996) 1-138; hep-th/9501144.

[7] A.B. Zamolodchikov, Sov. J. Nucl. Phys. 46 (1987) 1090.

[8] D.J. Amit, Y.Y. Goldschmidt, and G. Grinstein, J. Phys. 13 (1980) 585; C. Destri, Phys. Lett. B210 (1988) 173, Erratum: Phys. Lett. B213 (1988) 565; J. Balog and A. Hegedüs, Two-Loop Beta Functions of the Sine-Gordon Model, hep-th/0003258.

[9] J. F. Bennett and J. A. Gracey, Nucl.Phys. B563 (1999) 390, hep-th/9909046.

[10] M. Bershadsky, S. Zhukov and A. Vaintrob, Nucl.Phys. B559 (1999) 205, hep-th/9902180.

[11] N. Berkovits, C. Vafa and E. Witten, JHEP 9903 (1999) 018, hep-th/9902098.

[12] S. Guruswamy, A. LeClair and A. W. W. Ludwig, Nucl.Phys. B583 (2000) 475-512, cond-mat/9909143.

[13] J. M. Kosterlitz and D. J. Thouless, J. Phys. C6 (1973) 1181; J. M. Kosterlitz, ibid C7 (1971) 1016.

[14] R.M. Konik, H. Saleur, and A.W.W. Ludwig, Interplay of the Scaling Limit and the Renormalization Group: Implications for Symmetry Restoration, cond-mat/0009166.

[15] A. LeClair, Strong Coupling Fixed Points of Current Interactions and Disordered Fermions in 2D, condmat/0011413.

[16] B. Gerganov, A. LeClair and M. Moriconi, in preparation. 